\title{
All-Fiber-Based All-Normal Dispersion Supercontinuum Source Using a Femtosecond Fiber Laser with Hollow-Core Fiber Pulse Compression
}

\author{
I. B. Gonzalo ${ }^{1 *}$, T. Vestergaard ${ }^{2}$ and O. Bang ${ }^{1,2}$ \\ 1. DTU Fotonik, Department of Photonics Engineering, Technical University of Denmark, 2800 Kgs. Lyngby, Denmark \\ 2. NKT Photonics A/S, Blokken 84, DK-3460 Birkerød, Denmark. \\ *ibag@fotonik.dtu.dk
}

\begin{abstract}
We develop and thoroughly characterize an all-fiber-based all-normal dispersion supercontinuum source pumped with a femtosecond fiber laser at $1036 \mathrm{~nm}$ using hollow-core fiber pulse compression. Pulse length, supercontinuum spectrum, and noise are measured.

OCIS codes: (140.3510) Lasers, fiber; (320.5520) Pulse compression; (320.6629) Supercontinuum generation
\end{abstract}

\section{Introduction}

Fiber-based supercontinuum (SC) sources are of great interest for many applications as they offer broadband spectra, spatial coherence and high power spectral density [1]. In addition, low-noise SC can also be achieved with all-normal dispersion fibers (ANDi) under certain conditions [2]. For applications where broadband and low-noise fiber-based laser sources are needed, such as optical coherence tomography [3], the development of these SC sources are therefore needed. In this work, we present an all-fiber-based ANDi SC laser source pumped with a femtosecond fiber laser based on dispersive compression of a picosecond fiber laser using a hollow-core fiber (HCF) $[4,5]$. Characterization of the pump pulse length, SC spectrum, and noise of the SC source is carried out.

\section{Experimental setup}

The pump laser compression and SC generation setup is shown in Fig. 1 (a). Picosecond pump pulses with 2 W maximum average power at $1036 \mathrm{~nm}$ are delivered by a single-mode fiber (mode field diameter (MFD) is $15 \mu \mathrm{m}$ ) from the fiber laser (NKT Photonics). The pump pulse length at full width at half maximum (FWHM) is around 23 ps and was measured with an intensity autocorrelator (Femtochrome, FR-103HP). The bandwidth at $-10 \mathrm{~dB}$ is around $15 \mathrm{~nm}$, corresponding to a transform-limited pulse length of around $75 \mathrm{fs}$. By propagating the linearly chirped picosecond pulses along a 7-cell HCF (MFD = $7.8 \mu \mathrm{m}$ at $1036 \mathrm{~nm}$ ) shown in Fig. 1 (b), compression is achieved due to the positive dispersion of the fiber $(67 \mathrm{ps} / \mathrm{nm} / \mathrm{km}$ at $1036 \mathrm{~nm})$. The compression ratio depends on the HCF length. The HCF (Fig. 1 (d)) is designed to be single-mode, low-loss (66 dB/km at $1036 \mathrm{~nm}$ ) and polarization maintaining at the pump wavelength [6]. The power from the pump laser is maintained at its maximum in all the measurements, and the coupling efficiency (corrected by fiber attenuation) in the HCF is around $60 \%$, corresponding to a maximum of $1.2 \mathrm{~W}$ average power at the output of the HCF for the shortest HCF length. To estimate the fiber length needed for optimum compression, we solved the generalized nonlinear Schrodinger equation using the pump parameters, measured dispersion, and loss (Fig. 1 (d)). Simulations showed that transformlimited pulse length could be achieved with around $23 \mathrm{~m}$ long HCF, but the higher-order dispersion of the HCF limits the achievable compression [5]. Furthermore, spectral broadening in the HCF was not observed in the simulations during the compression process.

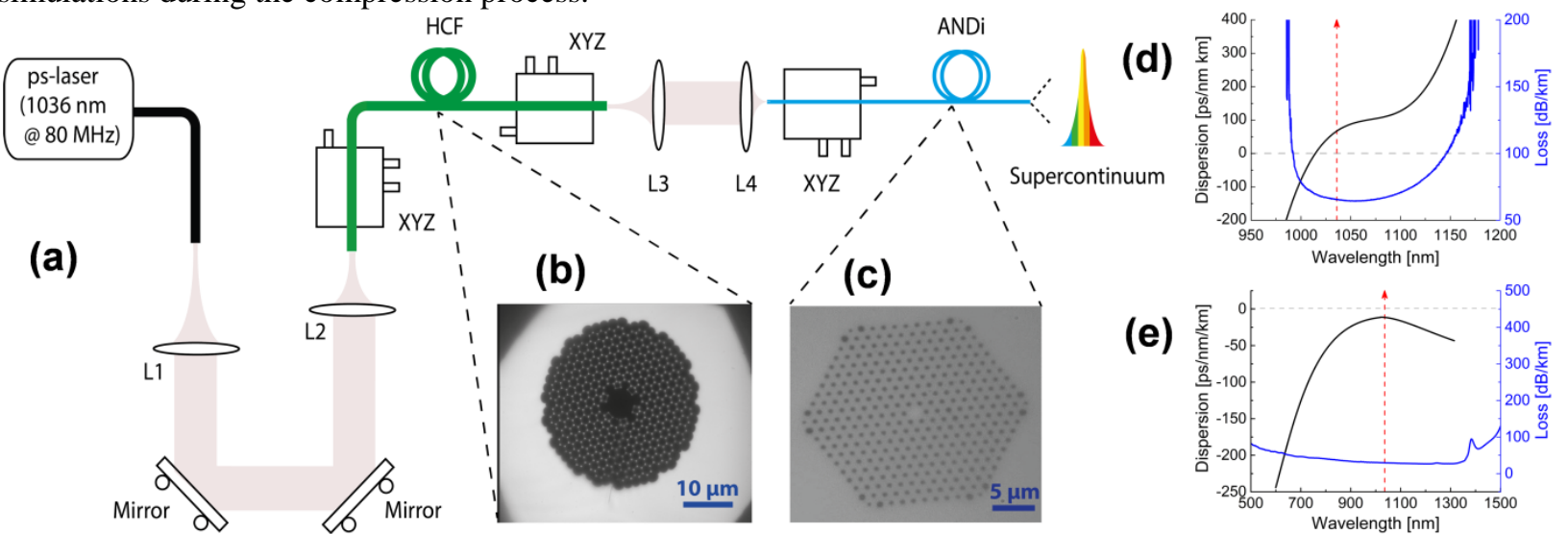

Fig. 1. (a) Sketch of the pump laser with HCF compression and ANDi SC generation setup. L1, L2, L3, L4-Lens, XYZ-3D Micrometer stage, HCF-Hollow-core fiber, ANDi-all-normal dispersion fiber. Microscope images of the end facet of the HCF (b) and ANDi (c) fibers. (d) and (e) are the measured dispersion (black) and loss (blue) of the HCF and ANDi fibers, respectively. Pump at $1036 \mathrm{~nm}$ is indicated by a red dashed line. 
After the pulse compression stage, the compressed pulses are coupled into a commercially available ANDi fiber (NL-1050-NEG-1 from NKT Photonics) for SC generation [2]. The average power at the output of the ANDi fiber is around $500 \mathrm{~mW}$. Figure 1 (c,e) shows the cross-section of the ANDi fiber and the measured dispersion and loss.

\section{Results}

The SC laser showed in Fig. 1 is thoroughly characterized by measuring the pulse length, SC spectrum, and noise as a function of the HCF length in a cutback experiment. The power from the picosecond pump laser is maintained constant and at maximum during the measurements. As in the simulations, no spectral broadening was measured after compression in the HCF. Pulse lengths of $255 \mathrm{fs}$ and $383 \mathrm{fs}$ at FWHM were measured for $26 \mathrm{~m}$ and $28 \mathrm{~m}$ long HCF (Fig. 2 (a)), respectively. The autocorrelation trace shows side lobes, meaning that the optimum compression is not fully completed. Also, the uncompensated higher order dispersion will play a role in the shape of the pulse [5]. The ANDi SC generated with picosecond and femtosecond pump pulses for a $9 \mathrm{~m}$ long HCF is shown in Fig. 2 (b). When the pump pulse length is 20 ps, only a Raman peak located at around $1086 \mathrm{~nm}$ is generated. Increasing the pump pulse length increases the peak power for a fixed average power of $1.2 \mathrm{~W}$, and thus the spectrum broadens only towards longer wavelengths due to the stimulated Raman scattering (SRS) [1]. For femtosecond pumping, the SC spectrum broadens symmetrically towards shorter and longer wavelengths than the pump, as expected for SC generated with ANDi fibers [2]. The spectrum is the broadest for the shortest femtosecond pump pulse, as shown in Fig. 2 (a). In addition to the spectral measurements, relative intensity noise (RIN) measurements of the ANDi SC were carried out by spectrally filtering the SC with $10 \mathrm{~nm}$ bandpass filters every $50 \mathrm{~nm}$, and detecting around 16000 pulses with a fast photodiode and an oscilloscope. The RIN is then calculated at each filtered wavelength as the ratio of the standard deviation to the mean of the distribution obtained from the maximum of the pulses. Preliminary RIN measurements are shown in Fig. 2 (c) using a $9 \mathrm{~m}$ and $0.5 \mathrm{~m}$ long ANDi fiber pumped with $255 \mathrm{fs}$ pulses. We observe that the noise of the ANDi SC generated with a $9 \mathrm{~m}$ long fiber is higher than with a $0.5 \mathrm{~m}$ long fiber for the measured spectral range. For instance, the RIN with the $0.5 \mathrm{~m}$ long fiber is always below $10 \%$, especially low for wavelengths longer than $1100 \mathrm{~nm}$, and higher than $10 \%$ for a $9 \mathrm{~m}$ long fiber. The excess noise observed for the $9 \mathrm{~m}$ long fiber could be associated, among other sources, to Raman noise generated when long ANDi fibers are used [2].
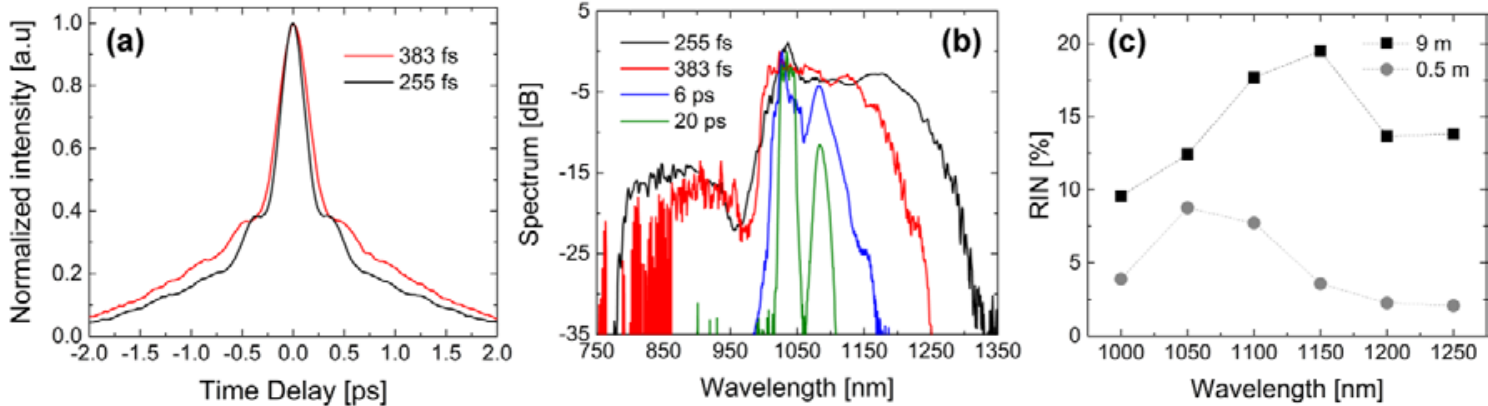

Fig. 2 (a) Measured pulse length at the output of the HCF for a $28 \mathrm{~m}$ (red) and $26 \mathrm{~m}$ (black) long HCF. The pulse lengths were calculated from the autocorrelation trace at FWHM by assuming Gaussian profile. (b) Measured SC spectra for femtosecond (black: 255 fs, red: 383 fs) and picosecond (blue: $6 \mathrm{ps}$, green: $20 \mathrm{ps)}$ pulses from the HCF and input average power of $1.2 \mathrm{~W}$. (c) RIN measurements for SC generated with $0.5 \mathrm{~m}$ (grey) and $9 \mathrm{~m}$ (black) long ANDi fiber pumped with 255 fs pulses.

\section{Conclusion}

In conclusion, we developed and characterized an all-fiber-based ANDi SC source pumped with pulses ranging from picoseconds to femtoseconds from a fiber laser compressed with a HCF. For different HCF lengths, the pulse length out of the HCF together with ANDi SC and RIN measurements were performed. Low-noise ANDi SC (RIN $<10 \%$ for wavelengths above the pump) was generated with the shortest pulse length, $255 \mathrm{fs}$, achieved in the experiment. The SC source presented here offers sufficiently broad spectrum and low-noise performance needed for OCT.

\section{References}

[1] J. M. Dudley, G. Genty, and S. Coen, "Supercontinuum generation in photonic crystal fiber," Rev. Mod. Phys. 78, 1135-1184 (2006).

[2] A. M. Heidt, J. S. Feehan, J. H. V. Price, and T. Feurer, “Limits of coherent supercontinuum generation in normal dispersion fibers,” J. Opt. Soc. Am. B 34, 764-775 (2017).

[3] M. Maria et al, “Q-switch-pumped supercontinuum for ultra-high resolution optical coherence tomography,” Opt. Lett. 42, 4744-4747 (2017). [4] D. Turchinovich, X. Liu, and J. Lægsgaard, "Monolithic all-PM femtosecond Yb-fiber laser stabilized with a narrow-band fiber Bragg grating and pulse-compressed in a hollow-core photonic crystal fiber," Opt. Express 16, 14004-14014 (2008).

[5] J. Lægsgaard and P. J. Roberts, “Dispersive pulse compression in hollow-core photonic bandgap fibers,” Opt. Express 16, $9628-9644$ (2008).

[6] M. Michieletto, J. K. Lyngsø, C. Jakobsen, J. Lægsgaard, O. Bang, and T. T. Alkeskjold, "Hollow-core fibers for high power pulse delivery,” Opt. Express 24, 7103-7119 (2016).

\section{Acknowledgement}

The authors acknowledge financial support from Innovationsfonden (SHAPEOCT-4107-00011B); GALAHAD Horizon 2020 Framework Programme (H2020) (732613) and Det Frie Forskningsråd (DFF) (LOISE-4184-00532B). 